\title{
OS EFEITOS DA ATIVIDADE FÍSICA NA DOENÇA DE ALZHEIMER
}

DOI: 10.48140/digitaleditora.2020.002.19

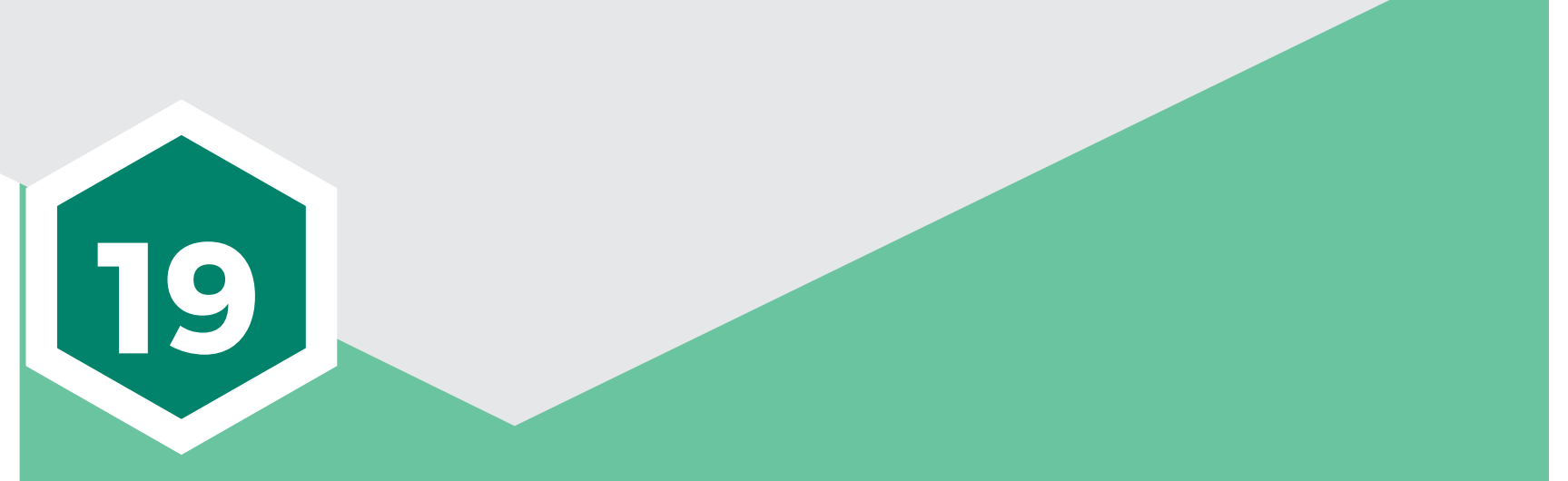

Robson Silva do prado

Graduando em Educação Física pela AESPI - Ensino Superior do Piauí

Teresina - Piauí

\section{iD https://orcid.org/0000-0002-7217-9535}

Rômulo Matos Pinheiro

Educador Físico, Especialista e Professor Auxiliar da Faculdade AESPI - Ensino Superior do Piauí

Teresina - Piauí

\section{iD https://orcid.org/0000-0002-9812-8170}

Wenderson Costa da Silva

Enfermeiro pelo Centro Universitário de Ciências e Tecnologia do Maranhão

Caxias- MA

\section{iD https://orcid.org/0000-0001-6031-9775}

Cristina Soares Oliveira

Discente de Psicologia pelo Centro Universitário de Ciências e Tecnologia do Maranhão

Caxias- MA

\section{iD https://orcid.org/0000-0002-4917-6811}

Eduardo Brito da Silva

Discente de Enfermagem pelo Centro Universitário de Ciências e Tecnologia do Maranhão

Caxias-MA

iD https://orcid.org/0000-0002-8571-7806
Rafael Andrade Da Silva

Enfermeiro pelo Centro universitário de Ciências e Tecnologia do Maranhão Caxias-MA

\section{iD https://orcid.org/0000-0002-0357-8102}

\section{Brunna Matos Sousa}

Enfermeira pelo Centro universitário de Ciências e Tecnologia do Maranhão Caxias-MA

\section{iD https://orcid.org/0000-0002-7009-6488}

Edilene Ferreira da Costa Santos

Discente de Psicologia pelo Centro Universitário de Ciências e Tecnologia do Maranhão Caxias-MA

\section{iD https://orcid.org/0000-0001-7304-9401}

Bianca Santos Soares

Enfermeira pelo Centro Universitário de Ciências e Tecnologia do Maranhão Caxias- MA

\section{iD https://orcid.org/0000-0003-0074-2082}

Cesar Augusto Pereira Souza Filho

Enfermeiro pelo Centro Universitário de Ciências e Tecnologia do Maranhão Caxias- MA.

iD https://orcid.org/0000-0002-5574-2736 


\section{OS EFEITOS DA ATIVIDADE FÍSICA NA DOENÇA DE ALZHEIMER}

DOI: $10.48140 /$ digitaleditora.2020.002.19

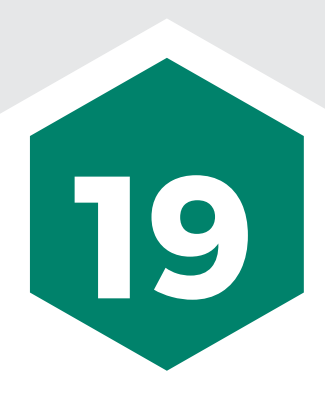

\section{RESUMO}

Objetivos: Avaliar os feitos da atividade física em indivíduos com Alzheimer.

Métodos: Trata-se de uma pesquisa bibliográfica do tipo revisão integrativa da literatura. Este procedimento foi escolhido por possibilitar a síntese e análise do conhecimento científico já produzido sobre o tema "os efeitos da atividade física na doença de alzheimer". Consultou-se por meio de descritores as bases de dados PubMed da National Library of Medicine; EMBASE (Biomedical answers) da ScienceDirect; e CINAHL (Cumulative Index to Nursing and Allied Health Literature).

Resultados: Nível de evidência foi considerado alto, sendo composto principalmente por ensaios clínicos randomizados (36,4\%\%), em que todos os estudos abordaram de forma quantitativa a análise dos dados (100,0\%). O grau de recomendação obteve-se maiores índices no A (72,7\%) para mudança na prática clínica. Os estudos tiveram como foco principal o impacto dos exercícios físicos na doença de Alzheimer, especialmente na função cognitiva; o perfil sociodemográfico das pessoas com Alzheimer que obtiveram mais benefícios na prática de atividade física; os exercícios físicos mais utilizados como forma de intervenção terapêutica; e a intensidade que esses exercícios devem ser realizados para trazerem um maior benefício.

Conclusão: A atividade física é uma importante intervenção complementar para indivíduos com doença de Alzheimer, pois os exercícios físicos tem demonstrado um potencial efeito na função cognitiva, linguagem, e nos movimentos corporais, reduzindo a progressão da doença, e proporcionando certa autonomia para os indivíduos com a doença de Alzheimer, bem como uma melhor qualidade de vida. 


\section{THE EFFECTS OF PHYSICAL ACTIVITY ON ALZHEIMER'S DISEASE}

DOI: 10.48140/digitaleditora.2020.002.19

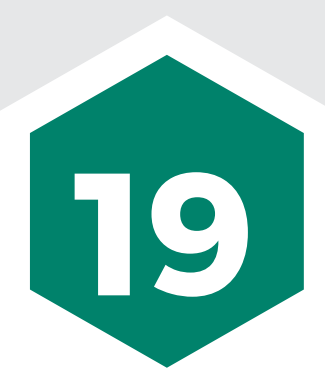

\section{ABSTRACT}

Recebido em:

Aprovado em:

Conflito de Interesse:

Suporte Financeiro: não houve
Objectives: Thus, the present study aims to analyze the response of the immune system to physical exercise at different intensities.

Methods: This is a bibliographic research study based on journals and scientific articles to evaluate the effect of the immune system on physical exercise in its different intensities, for the organization of this object of study, journals and articles subjects were read and interpreted in order to generate a clarification of the case under study, such as PubMed, Scielo. The study was carried out considering a search to present the importance on the subject through some studies from 5 years ago.

Results: Articles from journals with the effect of physical exercise in the immunological-co system were analyzed, where it was possible to analyze and observe that the immune system can have a beneficial according to the intensity applied.

Conclusion: It is worth considering in the prescription of the training to calculate and periodize the pre-scribed activity so that we can have a control of the intensity of physical exercise in rela-tion to the immune system. 


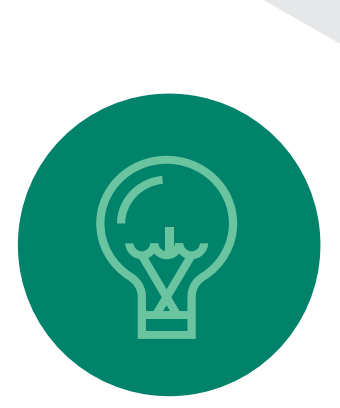

\section{INTRODUÇÃO}

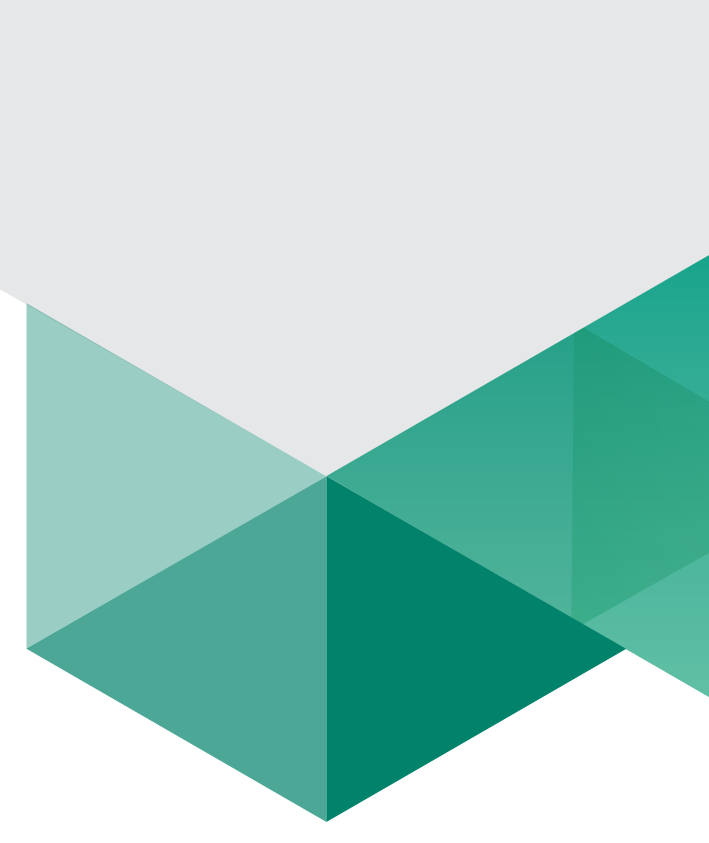

O rápido envelhecimento populacional em todo mundo, favorece o aumento de um grupo de doenças crônicas e degenerativas, tais doenças provocam danos severos em muitas habilidades corporais e cognitivas, prejudicando a qualidade de vida e proporcionando o sofrimento emocional nas pessoas acometidas como também em seus familiares e cuidadores (TALMELLI et al., 2013).

Dentre as doenças crônicas degenerativas a que atinge uma grande parcela da população idosa é a doença de Alzheimer (DA), que é definida como um transtorno neurodegenerativo progressivo, que provoca a deterioração da função da memória e também cognitiva, se manifesta através de uma variedade de sintomas com alterações comportamentais e neuropsiquiátricas (CONITEC, 2017).

Nos estudos de Teixeira et al. (2015) ao avaliarem os óbitos em idosos por doenças do sistema nervoso central no ano de 2009, na maioria dos casos avaliados a DA se apresentava como causa básica de óbitos, representando $65 \%$ das taxas de óbitos no público feminino e 51,1\% no público masculino.

A DA tem início lento e progressivo por vários anos, com alterações a nível bioquímico e neuropatológica divididas em mudanças estruturais que incluem alterações neurofibrilares, placas neuríticas e também mudanças no metabolismo amiloide, caracterizada pela morte neural e perdas sinápticas. $E$ as alterações nos sistemas neurotransmissores em que estas estão ligadas às mudanças estruturais (forma patológica) e desordenada na doença. Neste cenário alguns neurotransmissores são afetados totalmente ou parcialmente indicando um padrão de degeneração de sistemas, entretanto pode ocorrer dos sistemas neurotransmissores serem afetados em algumas áreas cerebrais, enquanto em outras áreas permanecem íntegras (BRASIL, 2013).

Para diagnóstico de Alzheimer não existe exames isolados, portanto é necessária uma triagem cognitiva e de alta sensibilidade para rastrear quadros iniciais de demência ou de leve comprometimento cognitivo. Entre tanto existe atualmente uma diversidade de exames laboratoriais de neuroimagem para investigação da DA. Alguns exames realizam a avaliação genética e análise de neurofilamentos presentes no líquido cefalorraquidiano, e conseguem diferenciar a DA de outras demências. Mas o diagnóstico precoce ainda é um desafio, por isso a importância da investigação clínica, que comtemple fatores epidemiológicos de risco para a doença, rastreio cognitivo de pacientes sob maior suspeita (APRAHAMIAN; MARTINELLI; YASSUDA, 2009).

Em relação ao tratamento da DA, este deve acontecer de forma multidisciplinar, pois a DA apre- 
senta diversos sinais e sintomas, necessitando de condutas especificas e adaptáveis ao estado de cada indivíduo. O tratamento é realizado de forma medicamentosas no intuito de estabilizar o comprometimento cognitivo, as alterações do comportamento e propiciar a realização das atividades do cotidiano com os mínimos efeitos adversos (CONITEC, 2017).

Nos estudos de Frota et al. (2016) destacam que ainda não existe estudos concretos o suficiente que demonstrem a eficiência das drogas atualmente em uso no tratamento de Alzheimer. Pois para elaboração dos fármacos leva-se em contas as hipóteses moleculares da DA, em que estas diferem quanto característica fisiopatológica, desta forma resultando em diferentes conclusões quanto ao efeito e também quanto a abordagem terapêutica.

Por isso a busca por intervenções não farmacológicas tem sido um assunto de interesse pelos pesquisadores, tendo em vista que as intervenções não-farmacológicas têm demonstrado resultados positivos no manejo de indivíduos com DA (VALE et al., 2011) Nesse contexto a prática regular de atividade física realizada de forma sistemática e com o acompanhamento profissional adequado tem apontado benefícios na estimulação cognitiva, e na preservação e até mesmo uma melhoria temporária de muitas funções cognitivas, na linguagem, e no comportamento, melhorando a qualidade de vida de indivíduos com DA (COELHO et al., 2009)

Diante do exposto elaborou-se a seguinte questão norteadora: Quais os efeitos da atividade física em indivíduos com Alzheimer? Para tal tendo como objetivo geral avaliar os feitos da atividade física em indivíduos com Alzheimer, e especificamente determinar o perfil sociodemográfico dos indivíduos beneficiados pela prática de atividade física; elencar os exercícios físicos utilizados como intervenção; e evidenciar a intensidade dos exercícios físicos que trazem maior benefício para os indivíduos com Alzheimer.

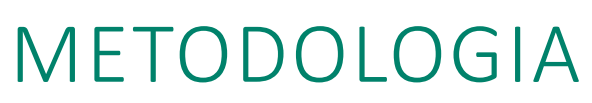

O presente estudo trata-se de uma pesquisa bibliográfica do tipo revisão integrativa da literatura. Este procedimento foi escolhido por possibilitar a síntese e análise do conhecimento científico já produzido sobre o tema "OS EFEITOS DA ATIVIDADE FÍSICA NA DOENÇA DE ALZHEIMER". Esta revisão utilizou a metodologia proposta no estudo de Oliveira et al. (2016).

A revisão integrativa da literatura é um método que visa sintetizar de forma abrangente os resultados da pesquisa de um determinado tema ou questão de forma sistemática, ordenada e ampla. É denominado integrativa porque fornece informações mais abrangentes sobre o assunto/problema. Dessa forma, os revisores/ pesquisadores podem conduzir revisões integrativas para diferentes propósitos e podem se concentrar em definições de conceitos, revisões teóricas ou análises metodológicas de tópicos específicos (ERCOLE; MELO; ALCOFORADO, 2014).

O tema determinou a construção da estratégia PICO, que representa um acrônimo para Paciente (P), Intervenção (I), Comparação (C) e Desfechos (O-outcomes), na qual foi utilizada para a geração da questão norteadora desta revisão integrativa da literatura: Quais os efeitos da atividade física na doença de Alzheimer? 
Para a localização dos estudos relevantes, que respondessem à pergunta de pesquisa, utilizou-se de descritores indexados nos idiomas português, inglês e espanhol. Os descritores foram obtidos a partir do Medical Subject Headings (MESH), dos Descritores em Ciências da Saúde (DeCS) e dos títulos CINAHL, como mostra o Quadro 1. Consultou-se por meio de descritores as bases de dados PubMed da National Library of Medicine; EMBASE (Biomedical answers) da ScienceDirect; e CINAHL (Cumulative Index to Nursing and Allied Health Literature).

Quadro 1. Elementos da estratégia PICO, descritores e palavras-chave utilizados. Teresina, PI, Brasil, 2020.

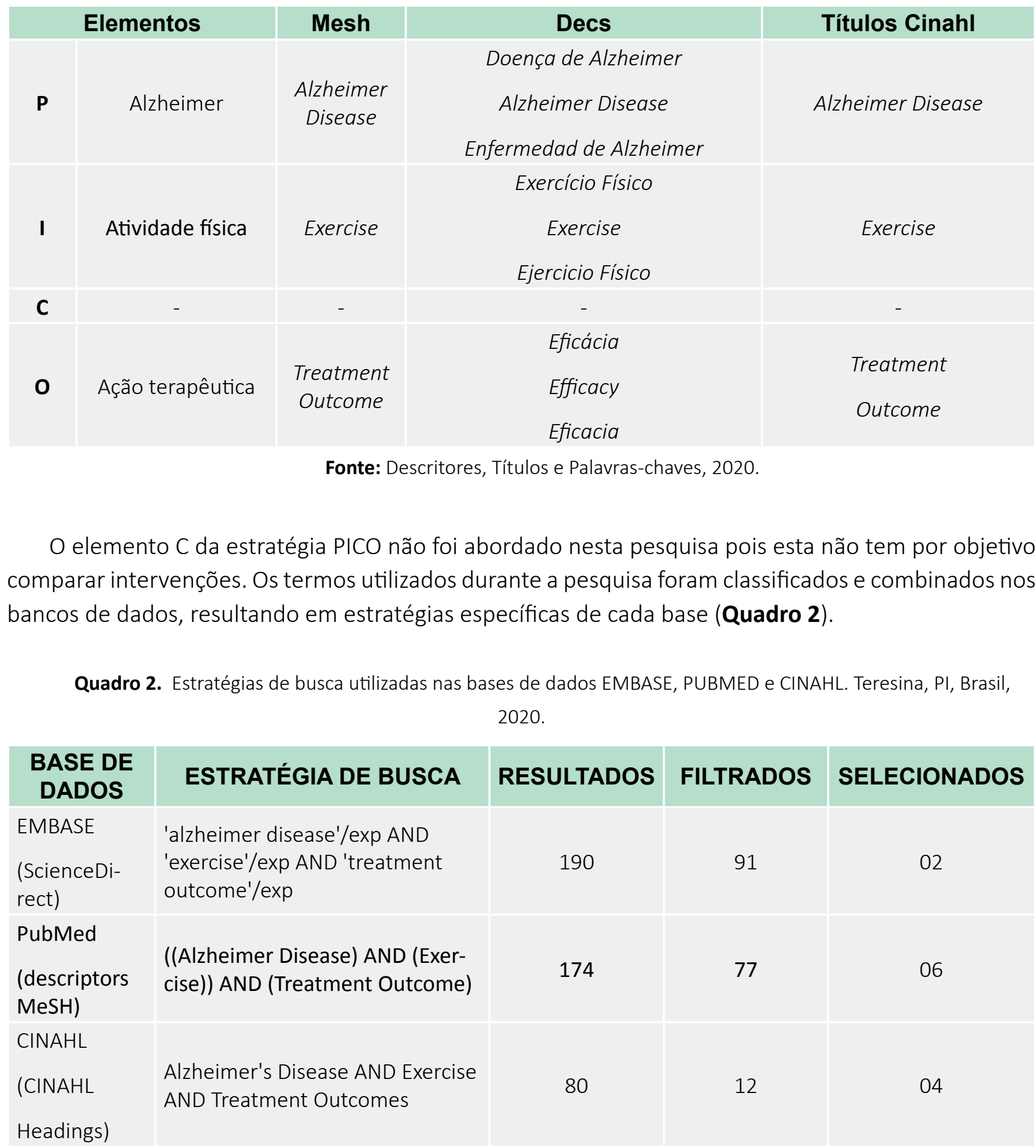

Fonte: Bases de dados, 2020. 
Como critérios de inclusão utilizaram-se estudos disponíveis em sua totalidade, publicados nos últimos cinco anos, de 2016 até 2020, nos idiomas Português, Espanhol e Inglês. Foram excluídos da busca inicial capítulos de livros, resumos, textos incompletos, teses, dissertações, monografias, relatos técnicos e outras formas de publicação que não artigos científicos completos.

A análise para seleção dos estudos foi realizada em duas fases, a saber:

Na primeira, os estudos foram pré-selecionados segundo os critérios de inclusão e exclusão e de acordo com a estratégia de funcionamento e busca de cada base de dados.

Na segunda fase os estudos foram analisados quanto ao potencial de participação no estudo, avaliando o atendimento à questão de pesquisa, bem como o tipo de investigação, objetivos, amostra, método, desfechos, resultados e conclusão, resultando em doze (12) artigos que atenderam a questão norteadora e forma adicionados ao estudo.

Quanto a análise e interpretação dos resultados, foram analisadas as informações coletadas nos artigos científicos e criadas categorias analíticas que facilitou a ordenação e a sumarização de cada estudo. Essa categorização foi realizada de forma descritiva, indicando os dados mais relevantes para o estudo.

A pesquisa levou em consideração os aspectos éticos da pesquisa quanto às citações dos estudos, respeitando a autoria das ideias, os conceitos e as definições presentes nos artigos incluídos na revisão. Optou-se pela análise em forma estatística e de forma de texto, utilizando cálculos matemáticos e inferências, que serão apresentados em quadros e tabelas para facilitar a visualização e compreensão.

As evidências científicas foram classificadas segundo os níveis e graus de recomendação propostos por Bork (2005), em que para níveis de evidências, temos: Nível 1 - Revisão sistemática; Nível 2 Ensaios clínicos randomizados; Nível 3 - Estudo de Coorte; Nível 4 - Estudo de Caso Controle; Nível 5 - Séries de casos; Nível 6 - Opiniões de especialistas; e Nível 7 - Estudos pré-clínicos (animais/in vitro). Para grau de recomendação: $\mathrm{A}$ - O resultado recomenda a intervenção; B - Resultado não é conclusivo (não é suficiente para confirmar a hipótese); e C- Resultado contraindica a intervenção.

Para ensaios clínicos não randomizados (estudo quase experimental), foi utilizado a Classificação proposta por Galvão (2006), o qual foi classificado como nível de evidência 3.

\section{RESULTADOS E DISCUSSÃO}

Encontrou-se cento e noventa (190) estudos como busca geral na EMABASE, e ao aplicar o filtro de estudos publicados nos últimos cinco anos, obteve-se noventa e um (91) estudos, destes foram analisados títulos e resumos, onde apenas dois (2) estudos foram condizentes com a questão desta pesquisa. Não foram encontrados filtros para "estudos realizados com seres humanos" e "Estudos completos". Entretanto, seguiu-se a mesma lógica de coleta de dados, como: estudos completos; publicados nos últimos cinco anos; realizados com humanos.

Na base PUBMED, como busca total foram encontrados cento e setenta e quatro (174) estudos, aplicando na pesquisa o filtro que limita por texto completo dos últimos cinco anos com humanos, obteve-se setenta e sete (77) estudos, destes foram analisados títulos e resumos e teve como resultado final de seis (6) estudos. 
$\mathrm{Na}$ CINAHL foram obtidos oitenta (80) estudos como busca geral, sendo que limitando a busca para artigos com texto completo realizado nos últimos cinco anos com humanos, obteve-se doze (12) estudos, sendo quatro (4) foram condizentes com a questão desta pesquisa após a análise dos títulos e resumos. Ao final, onze (11) estudos estavam de acordo com os objetivos propostos e foram incluídos nesta revisão.

O nível de evidência foi considerado alto, sendo composto principalmente por ensaios clínicos randomizados $(36,4 \% \%)$ e revisões sistemáticas $(27,3 \%)$, em que todos os estudos abordaram de forma quantitativa a análise dos dados $(100,0 \%)$. Com relação ao idioma houve prevalência do inglês (90,9\%), sendo os Estados Unidos e Dinamarca os países com mais estudos incluídos, com 27,3\% e $18,2 \%$, respectivamente. Com relação ao recorte temporal o ano de 2016 e 2019 foram os que mais forneceram evidências, com 36,4\% cada. O grau de recomendação obteve-se maiores índices no A (72,7\%) para mudança na prática clínica, conforme demostrado na Tabela 01. Os estudos avaliaram os efeitos da prática de atividade física sobre a doença de Alzheimer, como mostra o Quadro 3.

TABELA 01. Distribuição da caracterização das produções científicas revisadas. Teresina, PI, 2020. (n=11)

\begin{tabular}{|c|c|c|}
\hline Variáveis & $\mathbf{N}$ & $\%$ \\
\hline \multicolumn{3}{|l|}{ Abordagem do estudo } \\
\hline Quantitativo & 11 & 100,0 \\
\hline \multicolumn{3}{|l|}{ Delineamento da Pesquisa } \\
\hline Revisão sistemática & 03 & 27,3 \\
\hline Ensaio clínico randomizado & 04 & 36,4 \\
\hline Estudo quase experimental & 02 & 18,2 \\
\hline Estudo de Coorte & 01 & 9,1 \\
\hline Relato de Caso & 01 & 9,1 \\
\hline \multicolumn{3}{|l|}{ Nível de evidência } \\
\hline Nível 1 & 03 & 27,3 \\
\hline Nível 02 & 04 & 36,4 \\
\hline Nível 03 & 03 & 27,3 \\
\hline Nível 05 & 01 & 9,1 \\
\hline \multicolumn{3}{|l|}{ Grau recomendação } \\
\hline$A$ & 08 & 72,7 \\
\hline B & 03 & 27,3 \\
\hline Idioma & & \\
\hline
\end{tabular}




\begin{tabular}{|c|c|c|}
\hline Inglês & 10 & 90,9 \\
\hline Português & 01 & 9,1 \\
\hline \multicolumn{3}{|l|}{ Procedência } \\
\hline Estados Unidos & 03 & 27,3 \\
\hline Dinamarca & 02 & 18,2 \\
\hline Brasil & 01 & 9,1 \\
\hline Austrália & 01 & 9,1 \\
\hline China & 01 & 9,1 \\
\hline Finlândia & 01 & 9,1 \\
\hline Holanda & 01 & 9,1 \\
\hline Portugal & 01 & 9,1 \\
\hline \multicolumn{3}{|c|}{ Ano de Publicação } \\
\hline \multirow{2}{*}{2016} & 04 & 36,4 \\
\hline & 01 & 9,1 \\
\hline \multirow{2}{*}{2018} & 02 & 18,2 \\
\hline & 04 & 36,4 \\
\hline
\end{tabular}

Fonte: Dados da pesquisa, 2020.

Quadro 3. Publicações incluídas segundo objetivo principal, perfil amostral, intervenções/interesse e principais resultados. Teresina, PI, Brasil, 2020.

\begin{tabular}{|c|c|c|c|c|}
\hline $\begin{array}{c}\text { Autores/ } \\
\text { Ano }\end{array}$ & $\begin{array}{l}\text { Objetivo } \\
\text { principal }\end{array}$ & Perfil amostral & Intervenções/Interesse & Principais resultados \\
\hline $\begin{array}{l}\text { BRASURE } \\
\text { et al., } \\
2018\end{array}$ & $\begin{array}{l}\text { Avaliar a eficácia } \\
\text { da intervenção } \\
\text { da atividade físi- } \\
\text { ca em retardar o } \\
\text { declínio cognitivo } \\
\text { e atrasar o início } \\
\text { de comprometi- } \\
\text { mento cognitivo } \\
\text { e demência em } \\
\text { adultos sem } \\
\text { diagnóstico de } \\
\text { deficiências cog- } \\
\text { nitivas. }\end{array}$ & $\begin{array}{l}32 \text { estudos elegíveis } \\
\text { que compararam } \\
\text { intervenções usan- } \\
\text { do componentes de } \\
\text { atividade física com } \\
\text { um controle inativo } \\
\text { em adultos sem } \\
\text { diagnóstico de defi- } \\
\text { ciência cognitiva. }\end{array}$ & $\begin{array}{l}\text { Foram utilizados ensaios } \\
\text { publicados em inglês } \\
\text { que duraram } 6 \text { meses } \\
\text { ou mais, inscreveram } \\
\text { adultos sem prejuízos } \\
\text { cognitivos clinicamen- } \\
\text { te diagnosticados e } \\
\text { compararam desfechos } \\
\text { cognitivos e demências } \\
\text { entre intervenções de } \\
\text { atividade física e con- } \\
\text { troles inativos. Dos } 32 \\
\text { ensaios elegíveis, } 16 \\
\text { com baixo a moderado } \\
\text { risco de viés compara- } \\
\text { ram uma intervenção de } \\
\text { atividade física com um } \\
\text { controle inativo. }\end{array}$ & $\begin{array}{l}\text { As evidências foram insu- } \\
\text { ficientes para tirar con- } \\
\text { clusões sobre a eficácia } \\
\text { do treinamento aeróbio, } \\
\text { treinamento de resistência } \\
\text { ou tai chi para melhorar } \\
\text { a cognição. Evidências de } \\
\text { baixa resistência mostraram } \\
\text { que intervenções multi- } \\
\text { componentes de atividade } \\
\text { física não tiveram efeito na } \\
\text { função cognitiva. Evidências } \\
\text { de baixa resistência mos- } \\
\text { traram que uma interven- } \\
\text { ção de múltiplos domínios } \\
\text { compreendendo atividade } \\
\text { física, dieta e o treinamento } \\
\text { cognitivo melhorou vários } \\
\text { resultados cognitivos. }\end{array}$ \\
\hline
\end{tabular}




\begin{tabular}{|c|c|c|c|c|}
\hline $\begin{array}{l}\text { coX et } \\
\text { al., } 2019\end{array}$ & $\begin{array}{l}\text { Examinar a ade- } \\
\text { são e os resul- } \\
\text { tados de saúde } \\
\text { física em uma } \\
\text { intervenção de } \\
\text { AF domiciliar de } \\
24 \text { meses em } \\
\text { adultos mais } \\
\text { velho sem risco } \\
\text { de doença de } \\
\text { Alzheimer. }\end{array}$ & $\begin{array}{l}106 \text { homens e } \\
\text { mulheres, com } 60 \\
\text { anos ou mais, com } \\
\text { comprometimento } \\
\text { cognitivo leve ou } \\
\text { queixas de memó- } \\
\text { ria subjetiva e pelo } \\
\text { menos } 1 \text { fator para } \\
\text { risco cerebrovascu- } \\
\text { lar (inatividade físi- } \\
\text { ca, obesidade, hi- } \\
\text { pertensão, doença } \\
\text { cardíaca, diabetes } \\
\text { tipo II, tabagismo, } \\
\text { hipercolesterole- } \\
\text { mia). }\end{array}$ & $\begin{array}{l}\text { O pacote de intervenção } \\
\text { compreendeu três com- } \\
\text { ponentes: o programa } \\
\text { de atividade física, a } \\
\text { intervenção compor- } \\
\text { tamental e monitora- } \\
\text { mento de telefone. O } \\
\text { período de } 24 \text { meses } \\
\text { foi dividido em } 4 \text { etapas } \\
\text { - estágio 1: 0-6 meses; } \\
\text { Estágio 2: 6-12 meses; } \\
\text { Estágio 3: 12-18meses; } \\
\text { Estágio 4: 18-24 meses. }\end{array}$ & $\begin{array}{l}\text { A taxa de retenção de } 24 \\
\text { meses }(97,2 \%) \text { e a adesão } \\
\text { à atividade física mediana } \\
\text { de } 91,67 \% \text { (Q1 - Q3, 81,96, } \\
\text { 100,00) foram excelentes. } \\
\text { A longo prazo, o grupo de } \\
\text { intervenção alcançou me- } \\
\text { lhorias significativamente } \\
\text { melhores nos níveis de } \\
\text { atividade física, força das } \\
\text { pernas, massa gorda e dis- } \\
\text { tribuição de gordura em } \\
\text { relação ao controle. }\end{array}$ \\
\hline $\begin{array}{l}\text { ETNIER et } \\
\text { al., } 2018\end{array}$ & $\begin{array}{l}\text { Explorar a dife- } \\
\text { rença dos bene- } \\
\text { fícios cognitivos } \\
\text { alcançados em } \\
\text { resposta à ativi- } \\
\text { dade física como } \\
\text { uma função do } \\
\text { risco genético } \\
\text { de uma pessoa } \\
\text { para doença de } \\
\text { Alzheimer. }\end{array}$ & $\begin{array}{l}54 \text { Adultos mais } \\
\text { velhos (50-65 anos) } \\
\text { cognitivamente } \\
\text { normais com um } \\
\text { histórico familiar } \\
\text { para doença de } \\
\text { Alzheimer foram } \\
\text { recrutados em três } \\
\text { coortes para parti- } \\
\text { ciparem de um pro- } \\
\text { grama de atividade } \\
\text { física de } 8 \text { meses. }\end{array}$ & $\begin{array}{l}\text { Programa de atividade } \\
\text { física de pelo menos } 3 \\
\text { dias por semana por } \\
8 \text { meses. Cada sessão } \\
\text { consistia em exercícios } \\
\text { aeróbicos (caminhando } \\
\text { ao redor do perímetro } \\
\text { do ginásio por15-20 } \\
\text { min) e treinamento de } \\
\text { força por } 30-40 \text { min } \\
\text { (tempo aumentou ao } \\
\text { longo dos } 8 \text { meses). }\end{array}$ & $\begin{array}{l}\text { As melhorias na memória } \\
\text { foram associadas a partici- } \\
\text { pação no programa inde- } \\
\text { pendentemente do status } \\
\text { de portadora APOE } \varepsilon 4 .\end{array}$ \\
\hline $\begin{array}{l}\text { GROOT et } \\
\text { al., } 2016\end{array}$ & $\begin{array}{l}\text { Investigar o efei- } \\
\text { to das interven- } \\
\text { ções de atividade } \\
\text { física na função } \\
\text { cognitiva em } \\
\text { pacientes com } \\
\text { demência. }\end{array}$ & $\begin{array}{l}18 \text { estudos que fize- } \\
\text { ram ensaios de con- } \\
\text { trole randomizados } \\
\text { que aplicaram uma } \\
\text { intervenção de ati- } \\
\text { vidade física com } \\
\text { função cognitiva } \\
\text { como medida de } \\
\text { desfecho. }\end{array}$ & $\begin{array}{l}\text { Os escores de diferen- } \\
\text { ça média padronizada } \\
\text { pós-intervenção foram } \\
\text { computados para cada } \\
\text { estudo e combinados } \\
\text { em tamanhos de efei- } \\
\text { tos agrupados usando } \\
\text { meta-análise de efeitos } \\
\text { aleatórios. A análise } \\
\text { primária produziu um } \\
\text { efeito global positivo } \\
\text { das intervenções de } \\
\text { atividade física na fun- } \\
\text { ção cognitiva. Análises } \\
\text { secundárias revelaram } \\
\text { que as intervenções de } \\
\text { atividade física foram } \\
\text { igualmente benéficas } \\
\text { em pacientes com Al- } \\
\text { zheimer. }\end{array}$ & $\begin{array}{l}\text { Esta meta-análise sugere } \\
\text { que intervenções de ati- } \\
\text { vidade física influenciam } \\
\text { positivamente a função } \\
\text { cognitiva em pacientes } \\
\text { com demência. Esse efeito } \\
\text { benéfico foi independente } \\
\text { do diagnóstico clínico e da } \\
\text { frequência da intervenção, } \\
\text { sendo impulsionado por } \\
\text { intervenções que incluíram } \\
\text { exercício aeróbico. }\end{array}$ \\
\hline
\end{tabular}




\begin{tabular}{|c|c|c|}
\hline $\begin{array}{l}\text { HOF- } \\
\text { FMANN } \\
\text { et al., } \\
2016\end{array}$ & $\begin{array}{l}\text { Avaliar os efeitos } \\
\text { de um progra- } \\
\text { ma de exercício } \\
\text { aeróbico de } \\
\text { moderada a alta } \\
\text { intensidade em } \\
\text { pacientes com } \\
\text { doença de Al- } \\
\text { zheimer leve. }\end{array}$ & $\begin{array}{l}200 \text { pacientes com } \\
\text { doença de Alzhei- } \\
\text { mer leve. }\end{array}$ \\
\hline $\begin{array}{l}\text { JIA et al., } \\
2019\end{array}$ & $\begin{array}{l}\text { Estudar os efei- } \\
\text { tos de resposta à } \\
\text { dose de atividade } \\
\text { na cognição de } \\
\text { pacientes com } \\
\text { DA como uma } \\
\text { necessidade de } \\
\text { ser questão re- } \\
\text { solvida. }\end{array}$ & $\begin{array}{l}13 \text { estudos que } \\
\text { fizeram ensaios } \\
\text { clínicos randomiza- } \\
\text { dos foram incluídos } \\
\text { com uma amostra } \\
\text { de } 673 \text { indivíduos } \\
\text { com diagnóstico de } \\
\text { doença de Alzhei- } \\
\text { mer. }\end{array}$ \\
\hline $\begin{array}{l}\text { ÖHMAN } \\
\text { et al., } \\
2016\end{array}$ & $\begin{array}{l}\text { Examinar se um } \\
\text { programa regular } \\
\text { de exercícios de } \\
\text { longo prazo rea- } \\
\text { lizado por indiví- } \\
\text { duos com Alzhei- } \\
\text { mer em casa ou } \\
\text { como exercício } \\
\text { em grupo em } \\
\text { uma creche adul- } \\
\text { ta tem efeitos } \\
\text { benéficos na cog- } \\
\text { nição para exami- } \\
\text { nar os resultados } \\
\text { secundários de } \\
\text { um julgamento } \\
\text { que foi publicado } \\
\text { anteriormente. }\end{array}$ & $\begin{array}{l}210 \text { indivíduos com } \\
\text { doença de Alzhei- } \\
\text { mer e seus cuida- } \\
\text { dores conjugais } \\
\text { randomizados em } \\
\text { três grupos. }\end{array}$ \\
\hline
\end{tabular}

Foram recrutados 200 pacientes com doença de Alzheimer leve para um grupo de exercícios supervisionados (sessões de 60 minutos três vezes por semana durante 16 semanas) ou para um grupo controle.

Uma busca sistemática da literatura foi realizada para estudos elegíveis publicados até 01 de novembro de 2018 em três bancos de dados internacionais (PubMed, Cochrane Library e Embase) e dois bancos de dados chineses (Wanfang Data,China National Knowledge Infrastructure). Todas as análises foram conduzidas usando Stata 14.0. Devido à heterogeneidade entre os estudos, um modelo de efeitos aleatórios foi usado para esta meta-análise. Dois tipos de intervenção que compreendem exercícios personalizados domiciliares (HE) e exercício baseado em grupo (GE), cada uma duas vezes por semana durante um ano, foram comparados com um grupo controle (CG) que recebe cuidados habituais da comunidade. A função cognitiva foi medida utilizando-se o Teste de Desenho do Relógio (CDT), Fluência Verbal (VF), Classificação de Demência Clínica (CDR) e Miniexame de Estado Mental (MMSE) na linha de base e 3, 6 e 12 meses de seguimento.
A análise do grupo de intenção de tratar não mostrou diferenças significativas entre os grupos de intervenção e controle em mudança da linha de base do Teste de Modalidades de Dígito símbolo, outros testes cognitivos, qualidade de vida ou atividades de vida diária.

Os grupos de intervenção mostraram uma melhoria estatisticamente significativa na cognição dos indivíduos incluídos medidos pela pontuação do Miniexame do estado mental (SMD $=1,12 \mathrm{Cl}: 0,66 \sim 1,59)$ em comparação com os grupos de controle. As análises de subgrupo mostraram diferentes quantidades de atividade física e exercícios podem gerar efeitos diferentes.

A função executiva, medida por meio de CDT, melhorou no grupo HE, e as alterações na pontuação foram significativamente melhores do que as do CG aos 12 meses (ajustados por idade, sexo e $C D R, P=03$ ). Todos os grupos deterioraram-se no escore de Fluência Verbal e Miniexame de Estado Mental durante a intervenção, e não foram detectadas diferenças significativas entre os grupos no seguimento de 12 meses quando as análises foram ajustadas por idade, sexo e Classificação de Demência Clínica. 


\begin{tabular}{|c|c|c|c|c|}
\hline $\begin{array}{l}\text { PTOMEY } \\
\text { et al., } \\
2019\end{array}$ & $\begin{array}{l}\text { Avaliar a viabi- } \\
\text { lidade de uma } \\
\text { abordagem de } \\
\text { videoconferên- } \\
\text { cia em grupo } \\
\text { para aumentar } \\
\text { a intensidade de } \\
\text { atividade física } \\
\text { em adultos com } \\
\text { doença de Al- } \\
\text { zheimer e seus } \\
\text { cuidadores. }\end{array}$ & $\begin{array}{l}\text { Um total de nove } \\
\text { adultos com doen- } \\
\text { ça de Alzheimer } \\
\text { juntos com seus } \\
\text { cuidadores foram } \\
\text { matriculados no } \\
\text { estudo, e sete } \\
\text { ( } 78 \% \text { ) completaram } \\
\text { a intervenção de } 12 \\
\text { semanas. }\end{array}$ & $\begin{array}{l}\text { Sessões de } 30 \text { min de } \\
\text { duração por videocon- } \\
\text { ferência três vezes por } \\
\text { semana durante } 12 \\
\text { semanas para grupos de } \\
\text { cinco a oito díades cada } \\
\text { um em sua própria casa. }\end{array}$ & $\begin{array}{l}\text { A intensidade de atividade } \\
\text { física semanal aumentou } \\
\text { em adultos com doença } \\
\text { de Alzheimer ( } 49 \% \text { ) e cui- } \\
\text { dadores ( } 30 \%) \text {. O exercício } \\
\text { realizado por videocon- } \\
\text { ferência em grupo é uma } \\
\text { abordagem viável e po- } \\
\text { tencialmente eficaz para } \\
\text { aumentar a intensidade de } \\
\text { atividade física em adultos } \\
\text { com doença de Alzheimer e } \\
\text { seus cuidadores. }\end{array}$ \\
\hline $\begin{array}{l}\text { SAMPAIO } \\
\text { et al., } \\
2016\end{array}$ & $\begin{array}{l}\text { Analisar o efei- } \\
\text { to de uma in- } \\
\text { tervenção de } \\
\text { Treinamento } \\
\text { Multicomponen- } \\
\text { te (MT) sobre } \\
\text { função cognitiva, } \\
\text { aptidão funcional } \\
\text { e variáveis antro- } \\
\text { pométricas em } \\
\text { pacientes institu- } \\
\text { cionalizados com } \\
\text { Alzheimer. }\end{array}$ & $\begin{array}{l}37 \text { idosos institucio- } \\
\text { nalizados ( } 84,05 \pm \\
5,58 \text { anos) clinica- } \\
\text { mente diagnostica- } \\
\text { dos com doença de } \\
\text { Alzheimer (estágios } \\
\text { leves e moderados). }\end{array}$ & $\begin{array}{l}\text { Os pacientes foram di- } \\
\text { vididos em dois grupos: } \\
\text { Grupo Experimental } \\
\text { (GE, } n=19) \text { e Grupo } \\
\text { controle (GC, } n=18 \text { ). } \\
\text { O EG participou de um } \\
\text { programa de Treina- } \\
\text { mento Multicomponen- } \\
\text { te supervisionado de } \\
\text { seis meses (resistência } \\
\text { aeróbica, resistência } \\
\text { muscular, flexibilidade e } \\
\text { exercícios posturais) de } \\
\text { 45-55 minutos/sessão, } \\
\text { duas vezes/semana. }\end{array}$ & $\begin{array}{l}\text { Os resultados sugerem que } \\
\text { os programas de treinamen- } \\
\text { to multicomponente podem } \\
\text { ser uma importante estraté- } \\
\text { gia não farmacológica para } \\
\text { melhorar as funções físicas } \\
\text { e cognitivas em pacientes } \\
\text { com doença de Alzheimer } \\
\text { institucionalizados. }\end{array}$ \\
\hline $\begin{array}{l}\text { SANTIA- } \\
\text { GO et al., } \\
2016\end{array}$ & $\begin{array}{l}\text { Investigar os efei- } \\
\text { tos da atividade } \\
\text { física em pessoas } \\
\text { com Doença de } \\
\text { Alzheimer no for- } \\
\text { mato de estudo } \\
\text { de caso. }\end{array}$ & $\begin{array}{l}\text { Participaram deste } \\
\text { estudo dois sujei- } \\
\text { tos do projeto de } \\
\text { extensão Programa } \\
\text { de atividade física } \\
\text { para pessoas com } \\
\text { Doença de Alzhei- } \\
\text { mer. }\end{array}$ & $\begin{array}{l}\text { Consiste em atividades } \\
\text { físicas, realizadas du- } \\
\text { rante } 1 \text { hora, } 3 \text { vezes/ } \\
\text { semana. No exercício } \\
\text { agudo foi investigada } \\
\text { a frequência cardíaca } \\
\text { e pressão arterial an- } \\
\text { tes, durante e após a } \\
\text { sessão. Previamente a } \\
\text { entrada destes parti- } \\
\text { cipantes no projeto e } \\
\text { após } 4 \text { meses de partici- } \\
\text { pação foram realizadas } \\
\text { as seguintes avaliações: } \\
\text { massa corporal, estatu- } \\
\text { ra, minimental, flexibili- } \\
\text { dade e a escala de equi- } \\
\text { líbrio funcional (Berg). }\end{array}$ & $\begin{array}{l}\text { Demonstrou-se aumento } \\
\text { de } 36 \% \text { na frequência car- } \\
\text { díaca e } 15,2 \% \text { na pressão } \\
\text { arterial. Foram encontradas } \\
\text { alterações que apontam } \\
\text { para possível melhoria nas } \\
\text { funções cognitivas ( } 6,25 \%) \\
\text { e equilíbrio (3,1\%). Com } \\
\text { relação à flexibilidade, os } \\
\text { pacientes apresentaram } \\
\text { resultados opostos de au- } \\
\text { mento (37,1\%) e diminuição } \\
\text { (-3,6\%). }\end{array}$ \\
\hline
\end{tabular}




\begin{tabular}{|c|c|c|c|c|}
\hline $\begin{array}{l}\text { SOBOL et } \\
\text { al., } 2018\end{array}$ & $\begin{array}{l}\text { Investigar o } \\
\text { efeito do exer- } \\
\text { cício aeróbio de } \\
\text { intensidade mo- } \\
\text { derada-alta na } \\
\text { aptidão cardior- } \\
\text { respiratória, ou } \\
\text { seja, consumo de } \\
\text { oxigênio de pico } \\
\text { (Vo 2pico) de- } \\
\text { terminado pelo } \\
\text { teste de exercício } \\
\text { cardiopulmonar } \\
\text { direto respiração } \\
\text { a respiração, e2) } \\
\text { a associação en- } \\
\text { tre alterações no } \\
\text { vo 2pico e alte- } \\
\text { rações na cogni- } \\
\text { ção e sintomas } \\
\text { neuropsiquiátri- } \\
\text { cos em pacientes } \\
\text { com Doença de } \\
\text { Alzheimer leve. }\end{array}$ & $\begin{array}{l}55 \text { participantes } \\
\text { de três clínicas de } \\
\text { memória na área } \\
\text { metropolitana, com } \\
\text { idade entre 50-90 } \\
\text { anos, com diagnós- } \\
\text { tico de Doença de } \\
\text { Alzheimer de acor- } \\
\text { do com os critérios } \\
\text { NINCDS-ADRDA. }\end{array}$ & $\begin{array}{l}\text { Os participantes do gru- } \\
\text { po de intervenção exer- } \\
\text { citaram-se } 1 \text { h } 3 \text { vezes } \\
\text { semanalmente durante } \\
16 \text { semanas em grupos } \\
\text { de } 2-5 \text { participantes su- } \\
\text { pervisionados por um fi- } \\
\text { sioterapeuta experiente. } \\
\text { Os participantes do GC } \\
\text { receberam os cuidados } \\
\text { habituais durante as } 16 \\
\text { semanas da intervenção } \\
\text { de exercício. }\end{array}$ & $\begin{array}{l}\text { O consumo de oxigênio } \\
2 \text { pico aumentou } 13 \% \text { no } \\
\text { grupo de intervenção e } \\
\text { uma diferença entre os } \\
\text { grupos na mudança média } \\
\text { (3,92 ml / kg / min, IC 95\% } \\
6,34-1,51, \text { p = 0,003) esteve } \\
\text { presente a favor do grupo } \\
\text { de intervenção. Os dados } \\
\text { combinados de grupo de } \\
\text { intervenção e controle } \\
\text { mostraram associações po- } \\
\text { sitivas entre mudanças em } \\
\text { consumo de oxigênio } 2 \text { pico } \\
\text { e mudanças nos sintomas } \\
\text { neuropsiquiátrico (Rho = } \\
\text {-0,41, p = 0,042) e mudan- } \\
\text { ças na velocidade mental } \\
\text { e atenção (Rho = 0,36, p = } \\
0,010 \text { ), respectivamente. }\end{array}$ \\
\hline
\end{tabular}

Fonte: Dados da pesquisa, 2020.

Os estudos tiveram como foco principal o impacto dos exercícios físicos na doença de Alzheimer, especialmente na função cognitiva; o perfil sociodemográfico das pessoas com Alzheimer que obtiveram mais benefícios na prática de atividade física; os exercícios físicos mais utilizados como forma de intervenção terapêutica e a intensidade que esses exercícios devem ser realizados para trazerem maior benefício.

\section{EFEITOS DA ATIVIDADE FÍSICA NA DOENÇA DE ALZHEIMER}

O principal efeito relatado nos estudos foi a melhora da função cognitiva tanto em indivíduos com diagnóstico de doença de Alzheimer como em indivíduos com histórico familiar para a doença. Um programa de intervenção com diferentes tipos de atividades físicas auxilia na manutenção do desempenho cognitivo ao longo do tempo, contribuindo para redução do declínio da cognição e da demência e melhorando a memória (ETNIER et al., 2018; GROOT et al., 2016; JIA et al., 2019; SANTIAGO et al., 2016).

A realização de exercícios físicos melhora a circulação, amplia o fluxo sanguíneo e o fornecimento de oxigênio para o cérebro, promovendo maior vascularização, reduzindo a pressão arterial e os níveis de lipídios. Além disso, estimula a proliferação neuronal no hipocampo e amplifica a função do endotélio (ÖHMAN et al, 2016). Consequentemente, a elevação do fluxo sanguíneo desacelera o declínio cognitivo de forma direta ou de forma indireta, minimizando outros fatores de risco, como diabetes, obesidade e doenças cardiovasculares (BRASURE et al., 2018).

Entretanto, os estudos demonstraram que a melhora das funções cognitivas por meio da atividade física é mais observada em indivíduos com doença de Alzheimer em nível leve ou moderado, no estágio avançado o cérebro já se encontra muito danificado, fazendo com que os efeitos dos exercícios cheguem tarde demais (ÖHMAN et al., 2016; SAMPAIO et al., 2016). 
Os exercícios físicos funcionam como um modulador neuro protetor fundamental, realizando o controle da doença e aumentando a função cerebral de modo significativo (JIA et al., 2019). Assim, positivamente, a adoção de um programa de atividade física também atenua de forma geral os sintomas neuropsiquiátricos, inibindo ou atrasando o surgimento de uma neurose mais grave (HOFFMANN et al., 2016; SOBOL et al., 2018).

Outros efeitos relatados em indivíduos com doença de Alzheimer após a aplicação de intervenções regulares de atividade física foi a melhora e aumento da força dos membros inferiores, perda de massa gorda, ganho de massa magra e menores medidas de circunferência do quadril, que podem aparecer à curto prazo. À longo prazo é possível observar maior condicionamento físico, melhora da composição corporal, diminuição da sarcopenia e dos riscos de incapacidade (COX et al., 2019; SAMPAIO et al., 2016).

\section{PERFIL SOCIODEMOGRÁFICO MAIS BENEFICIADO PELA PRÁTICA DE ATIVIDADE FÍSICA}

Para avaliar com maior credibilidade o resultado das pesquisas torna-se necessário traçar um perfil, onde foi observado que, os pacientes com idades entre 50-90 anos diagnosticados com doença de Alzheimer e que tinham outras comorbidades controladas, puderam experimentar uma melhoria de seu condicionamento cardiorrespiratório além de um aumento da agilidade da memória e atenção (SOBOL et al., 2018).

Foi evidenciado ainda que os participantes que apresentavam demência em grau leve a moderado tiverem resultados mais positivos quando a sua saúde física, minimização de sintomas neuropsiquiátricos, prevenção de agravos e melhora/manutenção de suas funções cognitivas. O que ressalta a importância de estimular exercícios físicos e avaliar a intensidade correta para cada pessoa, independentemente da condição de demência (GROOT et al., 2016; HOFFMANN et al., 2016).

Quanto à prevalência do sexo dos indivíduos, foi observada a participação tanto de pessoas do sexo masculino como participantes do sexo feminino, ambos os gêneros se beneficiaram da realização de atividades físicas para a saúde física e mental e a redução de agravos. E foi possível perceber que, indivíduos com idades entre 53-84 anos mostraram melhor capitação de oxigênio e redução dos sintomas neuropsiquiátricos (SANTIAGO et al., 2016; SOBOL et al., 2018).

\section{OS PRINCIPAIS EXERCÍCIOS FÍSICOS UTILIZADOS COMO INTERVENÇÃO TERAPÊUTICA NA DOENÇA DE ALZHEIMER}

Uma vez que, a doença de Alzheimer afeta drasticamente o indivíduo e é gradual e progressiva, os estudos tem buscado formas de prevenção do agravo ou ainda, preservar/melhorar o desempenho cognitivo. Neste aspecto, a atividade física tem mostrado diversos benefícios aos pacientes que aderem à prática, destacando-se exercícios aeróbicos e treinamento de força, onde os mesmos mostraram-se capazes de acurar a memória de idosos que tinham a função cognitiva considerada normal, porém, apresentavam risco para o surgimento da enfermidade (ETNIER et al., 2018; SOBOL et al., 2018; HOFFMANN et al., 2016).

Segundo Groot et al. (2016), existe uma correlação entre a prática rotineira de exercícios físicos 
e a diminuição do risco de aparecimento de demência. Foi observado ainda que, tanto a prática de exercícios aeróbicos quanto realização de exercícios não aeróbicos produzem efeitos positivos sobre a saúde física e cognição dos indivíduos.

Não existe unanimidade entre os estudiosos a respeito dos exercícios físicos que provocam maiores benefícios para os pacientes, onde se deve levar em consideração ainda, o perfil individual do sujeito. Porém, foi observado que a realização de trilhas de caminhadas como atividade física de rotina pode promover uma melhora da função cognitiva, havendo a necessidade de se avaliar diferentes combinações de exercícios (JIA et al., 2019).

Corroborando com a afirmativa, o estudo de Öhman et al. (2016) afirma que a utilização de exercícios domiciliares personalizados, durante 1 ano (longo prazo), foi capaz de melhorar a função executora de idosos que apresentavam transtornos/distúrbios de memória, evidenciando a necessidade de avaliar o perfil dos pacientes de forma individual e investigar de forma mais profunda os mecanismos envolvidos para a estimulação de outras esferas da cognição.

A realização de atividades aeróbicas (leves), resistidas e de flexibilidade em associação também mostraram eficácia para a melhora, ainda que discreta, e a manutenção de funções físicas e cognitivas em indivíduos com doença de Alzheimer. Onde os benefícios adquiridos perduraram mesmo após o término do estudo e salientam a necessidade de mais pesquisas a fim de melhorar a qualidade de vida dessas pessoas (SANTIAGO et al., 2016).

\section{INTENSIDADE DOS EXERCÍCIOS FÍSICOS QUE TRAZEM MAIOR BENEFÍCIO PARA O INDIVÍDUO COM DOENÇA DE} ALZHEIMER

Não é possível determinar de forma geral qual intensidade de exercício é melhor e essa é a uma das principais dificuldades para prescrever atividade física às pessoas com doença de Alzheimer, pois a intensidade vai depender de diversos fatores, como idade, nível da doença e de comprometimento físico (SANTIAGO et al., 2016).

Embora não seja viável apontar qual intensidade de exercícios físicos promove maiores benefícios ao indivíduo com Alzheimer, os estudos afirmam que intervenções de alta frequência não necessariamente possuem maiores efeitos quando comparadas às intervenções de baixa frequência (GROOT et al., 2016; JIA et al., 2019).

Com relação ao tempo, quando comparadas, intervenções realizadas até duas horas por semana possuem maior eficácia que as realizadas por mais de duas horas durante o mesmo período; da mesma forma, as atividades feitas três vezes ou menos por semana e por mais de 16 semanas têm maior efeito que aquelas realizadas por mais de três vezes na semana e por menos de 16 semanas (JIA et al., 2019).

Sobre a função cognitiva, exercícios de menor intensidade parecem ter maior eficácia, porém apenas nos exercícios não aeróbicos (GROOT et al., 2016). O mais afirmado é que a prática de exercícios físicos de forma regular e personalizada promove efeitos positivos à pessoa com Alzheimer (ÖHMAN et al., 2016). 


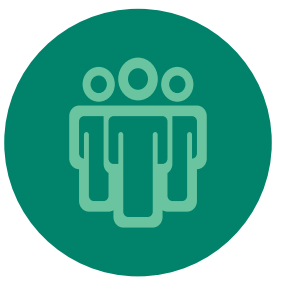

\section{CONCLUSÃO}

A doença de Alzheimer é uma patologia degenerativa e progressiva que afeta a população idosa, prejudicando diversas funções cerebrais o que acarreta em prejuízos cognitivos, comportamentais, e principalmente na redução da qualidade de vida, uma vez que o diagnóstico precoce é um grande desafio, a DA é uma das causas de óbitos mais frequente na terceira idade relacionada a doenças do sistema nervoso central.

Apesar do tratamento medicamentoso reduzir em parte o progresso da doença, deve se levar em conta outros fatores relacionado ao uso de medicamentos, tais como o tempo de tolerância do organismo, e os inúmeros efeitos adversos, além das características próprias e especificas da doença que cada indivíduo pode apresentar, por isso cada vez mais pesquisadores avaliam o potencial efeito de métodos alternativos e complementares ao tratamento medicamentoso, com destaque para a atividade física.

Sabe se que atividade física é um componente imprescindível para garantir a manutenção do organismo e prevenir uma variedade de doenças, neste sentido a atividade física seria uma importante intervenção complementar para indivíduos com DA, pois os exercícios físicos tem demonstrado um potencial efeito na função cognitiva, linguagem, e nos movimentos corporais, reduzindo a progressão da doença, e proporcionando certa autonomia para os indivíduos com DA bem como uma melhor qualidade de vida.

Ressalta-se ainda que atividade física pode ser utilizada tanto como intervenção preventiva, como também como intervenção complementar no tratamento da patologia, porém ainda são necessários mais estudos para compreender e delimitar a eficácia da atividade física na doença Alzheimer em mais de uma função cerebral que possa ser afetada bem como na condição física dos indivíduos. 


\section{REFERÊNCIAS}

APRAHAMIAN, I.; MARTINELLI, J.E.; YASSUDA, M.S. Doença de Alzheimer: Revisão da Epidemiologia e Diagnóstico. Rev Bras Clin Med. [S.I.], v. 7, p. 27-35, 2009.

BORK, A. M. T. Enfermagem baseada em evidencias. Rio de Janeiro: Guanabara Koonga, 2005.

BRASIL, Ministério da Saúde. Secretária de Atenção à Saúde. Portaria SAS/MS no 1.298, de 21 de novembro de 2013. Aprova o Protocolo Clínico e Diretrizes Terapêuticas da Doença de Alzheimer. Diário Oficial da União, Brasília, DF, 21 nov. 2013.

BRASURE, M. et al. Physical activity interventions in preventing cognitive declineand Alzheimer-Type Dementia. Annals of Internal Medicine, [S.I.], v. 168, n. 1, p. 30-41, jan. 2018.

COELHO, F.G.M et al. Atividade física sistematizada e desempenho cognitivo em idosos com demência de Alzheimer: uma revisão sistemática. Rev Bras Psiquiatr. v.31, n.2, p.163-70, 2009.

COMISSÃO NACIONAL DE INCORPORAÇÃO DE TECNOLOGIAS DO SUS (CONITEC). Protocolo Clínico e Diretrizes Terapêuticas para Doença de Alzheimer. Brasília: Ministério da Saúde, 2017.

COX, K. L. et al. A randomized controlled trial of adherence to a 24-month home-based physical activity program and the health benefits for older adults at risk of Alzheimer's disease: the AIBL active-study. Journal of Alzheimer's Disease, [S.I.], v. 70, p. 187-205, 2019.

ERCOLE, F. F.; MELO, L. S.; ALCOFORADO, C. L. G. C. Integrative review versus systematic review. Reme: Revista Mineira de Enfermagem, [S.I.], v. 18, n. 1, p. 9-11, 2014.

ETNIER, J. L. et al. The Physical Activity and Alzheimer's Disease (PAAD) Study: Cognitive outcomes. Annals of Behavioral Medicine, [S.I.], v. 52, n. 2, p. 175-185, fev. 2018.

FROTA, N.A.F. et al. Critérios para o diagnóstico de doença de Alzheimer. Dement Neuropsychol, [S.I.], v.5, n.1, p.5-10, 2011.

GALVÃO, C. M. Níveis de Evidência. Acta Paul Enferm, [S.I.], v. 19, n. 2, p. 5, 2006.

GROOT, G. et al. The effect of physical activity on cognitive function in patients with dementia: A meta-analysis of randomized control trials. Ageing Research Reviews, [S.I.], v. 25, p. 13-23, 2016.

HOFFMANN, K. et al. Moderate-to-High Intensity Physical Exercise in Patients with Alzheimer's Disease: A Randomized Controlled Trial. Journal of Alzheimer's Disease, [S.I.], v. 50, n. 2, p. 443-453, 2016.

JIA, R. et al. Effects of physical activity and exercise on the cognitive function of patients with Alzheimer disease: a meta-analysis. BCM Geriatrics, [S.I.], v. 19, n. 1, p. 181, jul. 2019.

ÖHMAN, H. et al. Efeitos do exercício sobre a cognição: o teste de exercício da doença de Alzheimer finlandês: um ensaio randomizado e controlado. Journal of the American Geriatrics Society, [S.I.], v. 64, n. 4, p. 731-738, 2016.

OLIVEIRA, F. B. M. et al. Relação entre a sobrecarga de trabalho e erros de administração de medicação na assistência hospitalar. Revista Ciências \& Saberes, Caxias, v. 2, n. 2. out./dez. 2016.

PTOMEY, L. T. et al. The feasibility of remotely delivered exercise session in adults with Alzheimer's disease and their caregivers. Journal of Aging and Physical Activity, [S.I.], v. 27, n. 5, p. 670-677, 2019. 
SAMPAIO, A. et al. Effects of a multicomponent exercise program in institutionalized elders with Alzheimer's disease. Sage Journals, [S.I.], v. 18, n. 2, p. 418-431.

SANTIAGO, A. M. et al. Efeitos da participação em programa de atividade física para pessoas com a doença de Alzheimer. Fisioterapia Brasil, [S.I.], v. 17, n. 3, p. 261-268, 2016.

SOBOL, N. A. et al. Change in Fitness and the Relation to Change in Cognition and Neuropsychiatric Symptoms After Aerobic Exercise in Patients with Mild Alzheimer's Disease. Journal of Alzheimer's Disease, [S.I.], v. 65, n. 1, p. $137-145,2018$

TALMELLI, L. F. S. et al. Doença de Alzheimer: declínio funcional e estágio da demência. Acta Paul Enferm. [S.I.], v.26, n.3, p.219-25, 2013.

TEIXEIRA, J. B. et al. Doença de Alzheimer: estudo da mortalidade no Brasil, 2000-2009. Cad. Saúde Pública, [S.I.], v. 31, n.4, p1-12, 2015.

VALE, F.A.C. et al. Tratamento da doença de Alzheimer. Dement Neuropsychol. v.5, n.1 (Suppl), p.34-48, 2011. 